\title{
Systematic Approaches for Blue Light-emitting Polymers by Introducing Various Naphthalene Linkages into Carbazole Containing PPV Derivatives
}

\author{
Taek $\mathrm{Ahn}^{\dagger}$ \\ Department of Chemistry, Kyungsung University, Busan 608-736, Korea
}

Received August 13, 2013; Revised August 23, 2013; Accepted August 28, 2013

Poly(2,3-naphthalenevinylene-alt-N-ethylhexyl-3,6-carbazolevinylene), 2,3-PNCPV, poly(2,6-naphthalene vinylenealt-N-ethylhexyl-3,6-carbazolevinylene), 2,6-PNCPV, and poly(1,4-naphthalenevinylene-alt-N-ethylhexyl-3,6carbazolevinylene), 1,4-PNCPV were synthesized through the Wittig polycondensation reaction. The conjugation lengths of the polymers were controlled by differently linked naphthalenes in the polymer main chain. The resulting polymers were completely soluble in common organic solvents, and exhibited good thermal stability at up to $400^{\circ} \mathrm{C}$. The synthesized polymers showed UV-visible absorbance and photoluminescence (PL) in the ranges of 357-374 $\mathrm{nm}$ and 487-538 nm, respectively. The carbazole and 2,3-linked naphthalene containing 2,3-PNCPV showed a blue PL peak at $487 \mathrm{~nm}$. A single-layer light-emitting diode was fabricated with an ITO/polymer/Al configuration. The electroluminescence (EL) emission of 2,3-PNCPV was shown at $483 \mathrm{~nm}$.

Keywords: Electroluminescence (EL), Poly(p-phenylenevinylene) (PPV), Naphthalene, Carbazole

\section{INTRODUCTION}

Since the first report on polymer light-emitting diodes (LEDs) based on poly( $p$-phenylenevinylene) (PPV), a number of conjugated backbone structures such as poly( $p$-phenylene) (PPP), polythiophene (PT) and polyfluorene (PF) have been shown to be important in realizing various emissive colors for polymeric LEDs [1-5]. A few polymers have been proven to be suitable for fabricating prototypes of LEDs which emit green, orange, or red light with high quantum efficiency and brightness. However, blue emission in polymeric materials remains an area of intense research, in order to achieve competiveness with newly developed GaN-based inorganic LEDs. The feasibility and processability of large-scale applications using polymeric materials has spurred research into the use of conjugated polymers in blue light emission. Blue EL has been observed from partially eliminated PPV, poly( $p$-phenylene)

${ }^{\dagger}$ Author to whom all correspondence should be addressed: E-mail: taekahn@ks.ac.kr

\section{Copyright $@ 2013$ KIEEME. All rights reserved.}

(PPP), poly(alkylfluorene), and many types of copolymers, but these polymers have still shown limited processability and mechanical properties in devices [6-10]. The search for a stable, highly efficient, and pure-blue light-emitting polymer still continues.

In a previous report, an attempt was made to control the emission color by inserting differently linked biphenyl units, and almost pure blue emission was obtained from the copolymer between carbazole and the 3,3'-linked biphenyl unit [11]. It was found that the copolymerization of carbazole and differently linked biphenyl groups enables the tenability of electronic properties such as the emission color.

Based on these considerations, three alternating copolymers have been synthesized based on carbazole and 2,3 (2,6 or 1,4)-linked naphthalene units through Wittig condensation polymerization. The simple concept of designing carbazole and naphthalene-containing copolymers is to introduce differently linked naphthalene units. This may provide an effective approach to tailoring the spectral characteristics to obtain blue light emission. The synthetic routes and polymer structures are shown in Scheme 1. 


\section{EXPERIMENTS}

Materials. 2,3-Dimethylnaphthalene, 2,6-dimethylnaphthalene, 1,4-dimethylnaphthalene, carbazole, 2-ethylhexylbromide, $\mathrm{N}$-bromosuccinimide (NBS), triphenylphosphine $\left(\mathrm{PPh}_{3}\right)$, potassium $t$-butoxide from Aldrich Chemical Co. were used without any further purification. $N, N$-Dimethylformamide (DMF), tetrahydrofuran (THF), carbon tetrachloride $\left(\mathrm{CCl}_{4}\right)$, ethanol, methanol, and chloroform were obtained from Junsei Chemical Co. and also used without purification.

Instrumentation. ${ }^{1} \mathrm{H}$ NMR spectra were recorded with a Bruker AMX $300 \mathrm{MHz}$ spectrometer. The UV-visible spectra of the 2,3-PNCPV, 2,6-PNCPV and 1,4-PNCPV were measured on a Shimadzu UV-3100S. The FT-IR spectra were measured by an EQUINOX 55 spectrometer. Thermogravimteric analyses (TGA) and differential scanning calorimetry (DSC) measurements of the polymers were performed under nitrogen atmosphere at a heating rate of $10{ }^{\circ} \mathrm{C} / \mathrm{min}$ with Dupont 9900 analyzers. The photoluminescence spectra of the polymers were obtained using a Spex Fluorolog-3 (model FL3-11) spectrofluorometer. The EL spectra of LED devices were measured with a Minolta CS-1000 spectroradiometer. Luminance-current-voltage (L-I-V) characteristics were recorded using a programmable current/voltage source (Keithley 238) and a luminance meter (Minolta LS-100).

Diphosphonium Salts Monomers. 2,3-Bis(bromomethyl) naphthalene (1). To synthesize compound $1,1.47 \mathrm{~g}(8.0 \mathrm{mmol})$ of $N$-bromosuccinimide (NBS) was dissolved in $50 \mathrm{~mL}$ of $\mathrm{CCl}_{4}$ under nitrogen atmosphere. Then $0.62 \mathrm{~g}$ ( $4.0 \mathrm{mmol})$ of 2,3-dimethylnaphthalene and a catalytic amount of benzoyl peroxide (BPO) were added to the solution, which was then heated to $90^{\circ} \mathrm{C}$ for $3 \mathrm{hr}$ under nitrogen atmosphere. The completion of the reaction was identified by the appearance of succinimide on the surface of the reaction solution. The solution was extracted with methylene chloride and $\mathrm{NaOH}$ solution. The organic layer was washed with water and dried over anhydrous $\mathrm{MgSO}_{4}$. The solvent was then removed by distillation to give $0.9 \mathrm{~g}(71.4 \%)$ of white solid. ${ }^{1} \mathrm{H}$ NMR $\left(\mathrm{CDCl}_{3}\right.$, ppm) $\delta 8.01 \sim 7.42$ (m, 6H, aromatic), 4.69 (s, $4 \mathrm{H},-\mathrm{CH}_{2} \mathrm{Br}$ ). Anal. Calcd. for $\mathrm{C}_{12} \mathrm{H}_{10} \mathrm{Br}_{2}$ : C, 45.90; H, 3.21. Found: $\mathrm{C}$, 45.80; H, 3.31 .

2,3-Naphthalenebis(triphenylphosphonium bromide) (2). A solution of $1.0 \mathrm{~g}(3.2 \mathrm{mmol})$ of compound $\mathbf{1}$ and $1.83 \mathrm{~g}(7.0$ $\mathrm{mmol}$ ) of triphenylphosphine in $20 \mathrm{~mL}$ of DMF was stirred and reacted at room temperature for $12 \mathrm{hr}$. The resulting mixture was poured into diethyl ether. After filtration and vacuum drying, diphosphonium salt monomer, compound 2, was obtained as a white solid. The product yield was $2.1 \mathrm{~g}(78 \%) .{ }^{1} \mathrm{H} \mathrm{NMR}\left(\mathrm{CDCl}_{3}\right.$ , ppm) $\delta$ 7.89-7.43 (m, $36 \mathrm{H}$, aromatic $\mathrm{H}), 5.28\left(\mathrm{~d}, 4 \mathrm{H},-\mathrm{PCH}_{2}-\right)$. Anal. Calcd. for $\mathrm{C}_{48} \mathrm{H}_{40} \mathrm{Br}_{2} \mathrm{P}_{2}$ : C, 68.75; H, 4.81. Found: C, 68.10; H, 4.51 .

2,6-Bis(bromomethyl)naphthalene (3). To synthesize compound $3,1.47 \mathrm{~g}(8.0 \mathrm{mmol})$ of $\mathrm{N}$-bromosuccinimide (NBS) was dissolved in $50 \mathrm{~mL}$ of $\mathrm{CCl}_{4}$ under nitrogen atmosphere. Then 0.62 $\mathrm{g}(4.0 \mathrm{mmol})$ of 2,6-dimethylnaphthalene and a catalytic amount of benzoyl peroxide (BPO) were added to this solution and the following synthetic procedures were performed in the same manner as for compound 1 . The product yield was $0.8 \mathrm{~g}(63.5 \%)$. ${ }^{1} \mathrm{H} \mathrm{NMR}\left(\mathrm{CDCl}_{3}\right.$, ppm) $\delta$ 7.96-7.29 (m, 6H, aromatic), $4.62(\mathrm{~s}, 4 \mathrm{H}$, $-\mathrm{CH}_{2} \mathrm{Br}$ ). Anal. Calcd. for $\mathrm{C}_{12} \mathrm{H}_{10} \mathrm{Br}_{2}$ : C, 45.90; H, 3.21. Found: C, $45.50 ; \mathrm{H}, 3.25$.

2,6-Naphthalenebis(triphenylphosphonium bromide) (4). A solution of $1.0 \mathrm{~g}$ ( $3.2 \mathrm{mmol})$ of compound 3 and $1.83 \mathrm{~g}$ (7.0 mmol) of triphenylphosphine in $20 \mathrm{~mL}$ of DMF was stirred and reacted at room temperature for $12 \mathrm{hr}$. The following synthetic procedures were performed in the same manner as for compound 2. The product yield was $1.9 \mathrm{~g}(70.6 \%) .{ }^{1} \mathrm{H} \mathrm{NMR}\left(\mathrm{CDCl}_{3}\right.$,

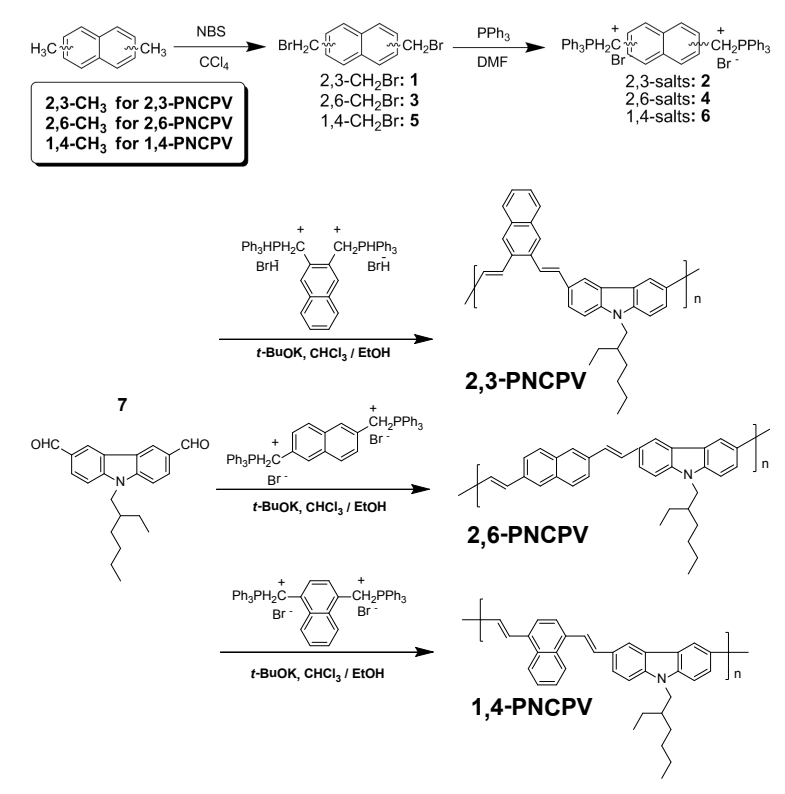

Scheme 1. Synthetic routes and polymer structures of 2,3-PNCPV, 2,6-PNCPV and 1,4-PNCPV.

ppm) $\delta$ 7.96-7.39 (m, $36 \mathrm{H}$, aromatic $\mathrm{H}), 5.26\left(\mathrm{~d}, 4 \mathrm{H},-\mathrm{PCH}_{2}{ }^{-}\right)$. Anal. Calcd. for $\mathrm{C}_{48} \mathrm{H}_{40} \mathrm{Br}_{2} \mathrm{P}_{2}$ : C, 68.75; H, 4.81. Found: $\mathrm{C}, 68.20 ; \mathrm{H}$, 4.49 .

1,4-Bis(bromomethyl)naphthalene (5). To synthesize compound $5,1.47 \mathrm{~g}(8.0 \mathrm{mmol})$ of $\mathrm{N}$-bromosuccinimide (NBS) was dissolved in $50 \mathrm{~mL}$ of $\mathrm{CCl}_{4}$ under nitrogen atmosphere. Then 0.62 $\mathrm{g}(4.0 \mathrm{mmol})$ of 1,4-dimethylnaphthalene and a catalytic amount of benzoyl peroxide (BPO) were added to this solution and the following synthetic procedures were performed in the same manner as for compound $\mathbf{1}$. The product yield was $0.7 \mathrm{~g}(55.6 \%)$. ${ }^{1} \mathrm{H}$ NMR $\left(\mathrm{CDCl}_{3}\right.$, ppm) $\delta$ 7.92-7.30 (m, 6H, aromatic), 4.60 (s, $4 \mathrm{H}$, $-\mathrm{CH}_{2} \mathrm{Br}$ ). Anal. Calcd. for $\mathrm{C}_{12} \mathrm{H}_{10} \mathrm{Br}_{2}$ : C, 45.90; H, 3.21. Found: C, 45.30; H, 3.15.

1,4-Naphthalenebis(triphenylphosphonium bromide) (6). A solution of $1.0 \mathrm{~g}$ ( $3.2 \mathrm{mmol})$ of compound $\mathbf{5}$ and $1.83 \mathrm{~g}$ (7.0 mmol) of triphenylphosphine in $20 \mathrm{~mL}$ of DMF were stirred and reacted at room temperature for $12 \mathrm{hr}$. The following synthetic procedures were performed in the same manner as for compound 2. The product yield was $2.1 \mathrm{~g}(78.0 \%) .{ }^{1} \mathrm{H} \mathrm{NMR}\left(\mathrm{CDCl}_{3}\right.$, ppm) $\delta 7.94 \sim 7.31(\mathrm{~m}, 36 \mathrm{H}$, aromatic $\mathrm{H}), 5.22\left(\mathrm{~d}, 4 \mathrm{H},-\mathrm{PCH}_{2}{ }^{-}\right)$. Anal. Calcd. for $\mathrm{C}_{48} \mathrm{H}_{40} \mathrm{Br}_{2} \mathrm{P}_{2}$ : C, 68.75; H, 4.81. Found: C, 68.55; H, 4.75 .

Dialdehyde Monomers. 9-(2-Ethylhexyl)carbazole-3,6-dicarbaldehyde (7). Compound 7 was synthesized by an adapted literature procedure presented in previous work [10].

Polymerization of $2,3(2,6$ or $\mathbf{1 , 4})$-PNCPV. A solution of 0.39 g (1.19 mmol) of 9-(2-ethylhexyl)carbazole-3,6-dicarbaldehyde and $1 \mathrm{~g}(1.19 \mathrm{mmol})$ of each diphosphonium salt monomer (2,3-naphthalenelbis(triphenylphosphonium bromide) for 2,3-PNCPV, 2,6-naphthalenebis(triphenylphosphonium bromide) for 2,6-PNCPV or 1,4-naphthalenebis (triphenylphosphonium bromide) for 1,4-PNCPV) was prepared in $10 \mathrm{~mL}$ of chloroform. In $10 \mathrm{~mL}$ of ethanol, $0.67 \mathrm{~g}(6 \mathrm{mmol})$ of potassium $t$ butoxide was dissolved completely, and this base solution was carefully dropped into the solution of monomers. After 1-2 days, the polymer product was precipitated from methanol. The crude polymers were filtered and then purified using a Soxhlet extractor in methanol for 2 days. The polymer yields were $0.35 \mathrm{~g}(62.5 \%)$ for 2,3-PNCPV, $0.31 \mathrm{~g}$ (55.4\%) for 2,6-PNCPV and $0.33 \mathrm{~g}$ (58.9\%) for 1,4-PNCPV. 


\section{RESULTS AND DISCUSSION}

All of the synthesized polymers were highly soluble in common organic solvents such as tetrahydrofuran, chloroform, methylene chloride, and 1,2-dichloroethane. They could be spincast onto various substrates to give highly homogeneous thin films without heat treatment. The polymerization results of the polymers synthesized are summarized in Table 1 . The numberaverage molecular weight $\left(M_{\mathrm{n}}\right)$ and the weight-average molecular weight $\left(M_{\mathrm{w}}\right)$ of the polymers, determined by gel permeation chromatography using polystyrene standards, were in the range of 4,100-5,600 and 7,300-8,900, respectively, with a polydispersity index (PDI) of 1.6-2.1.

Figure 1 shows the typical FT-IR spectra of 2,3-PNCPV, 2,6-PNCPV and 1,4-PNCPV. All polymers show weak but clear absorption peaks at about $960 \mathrm{~cm}^{-1}$ corresponding to the out-ofplane bending mode of the trans-vinylene. This proves that the vinylene double bond formation and the consequent polymerization reaction were successful $[12,13]$.

The thermal properties of the synthesized polymers were evaluated by means of TGA and DSC under nitrogen atmosphere. All synthesized polymers showed good thermal stability up to $400^{\circ} \mathrm{C}$ in TGA thermograms as shown in Fig. 2. The inset of Fig. 2 shows the DSC thermograms of polymers of 2,6-PNCPV and 1,4-PNCPV, 2,6-PNCPV shows higher $T_{\mathrm{g}}$ at about $137^{\circ} \mathrm{C}$ than 1,4-PNCPV $\left(109^{\circ} \mathrm{C}\right)$. The lower $T_{\mathrm{g}}$ of 1,4-PNCPV might be caused by the asymmetric naphthalene linkage.

Figure 3 shows the UV absorption spectra of 2,3-PNCPV, 2,6-PNCPV and 1,4-PNCPV in a thin film on a quartz plate. The maximum UV-visible absorptions of 2,6-PNCPV and 1,4-PNCPV appear at approximately 374 and $369 \mathrm{~nm}$, respectively. The 2,3-PNCPV film shows the most blue-shifted absorption maximum and edge at 357 and $453 \mathrm{~nm}$, respectively. This indicates that the $\pi$-electron delocalization of the polymer containing a 2,3-linked naphthalene unit is interrupted to a greater extent than that in the polymer containing 2,6- and 1,4-linked naphthalene units. All carbazole-containing copolymers additionally showed two absorption peaks at about 300 and $250 \mathrm{~nm}$ resulting from the carbazole unit in each polymer.

Figure 4 shows the PL spectra recorded using excitation wavelengths corresponding to the absorption maximum of each polymer in film. The PL spectra showed definite changes in emission color due to the naphthalene linkages in the polymer main chain. The spectra of the film samples resemble those of the corresponding solutions (not shown here), but are shifted to a longer wavelength. The 1,4-PNCPV film shows a maximum emission peak at about $538 \mathrm{~nm}$. But, the 2,6-PNCPV film shows a more blue-shifted PL emission maximum than 1,4-PNCPV at 504 $\mathrm{nm}$. The far more bent-type 2,3-PNCPV polymer exhibits almost pure blue light emission at $487 \mathrm{~nm}$. The reason for the blue-shift in the emission of 2,3-PNCPV compared with 2,6-PNCPV and 1,4-PNCPV is the same that for the UV-visible absorption of the polymers.

All polymers are highly fluorescent both in solution and film. The quantum efficiency of each polymer in chloroform solution was determined two or more times using a dilute quinine sulfate as a standard $\left(\sim 1 \times 10^{-5} \mathrm{M}\right.$ solution in $\left.0.10 \mathrm{M} \mathrm{H}_{2} \mathrm{SO}_{4}\right)$ [14]. The measured values were then averaged. The PL quantum yields of 2,3-, 2,6- and 1,4-PNCPV were determined as $\Phi_{\text {sol }}=0.72,0.69$ and 0.63 which are comparable values to other alkoxy or alkyl substituted PPV derivatives. Considering the utility of these compounds for applications in thin-film displays, the film PL quantum efficiency is more meaningful than the solution value. The film PL efficiencies were measured using an optically dense configuration of diphenylanthracene as the standard (dispersed
Table 1. Polymerization results of 4,4'(3.3')-PBPMEH-PPV and 4,4'(3.3')-PBPCAR-PPV .

\begin{tabular}{cccccc}
\hline polymers & Yield (\%) & $\mathrm{M}_{\mathrm{n}}$ & $\mathrm{M}_{\mathrm{w}}$ & PDI & $\mathrm{T}_{\mathrm{g}}\left({ }^{\circ} \mathrm{C}\right)$ \\
\hline \hline 2,3-PNCPV & 62.5 & 4,200 & 8,700 & 2.1 & - \\
\hline 2,6-PNCPV & 55.4 & 4,100 & 7,300 & 1.8 & 137 \\
\hline 1,4-PNCPV & 58.9 & 5,600 & 8,900 & 1.6 & 109 \\
\hline
\end{tabular}

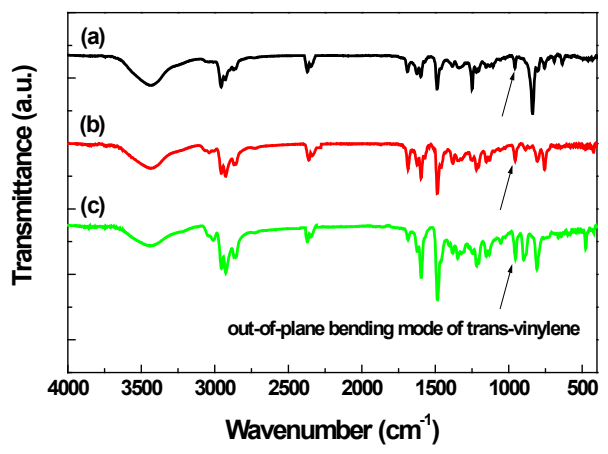

Fig. 1. FT-IR spectra of (a) 2,3-PNCPV, (b) 2,6-PNCPV, and (c) 1,4-PNCPV in KBr pellets.

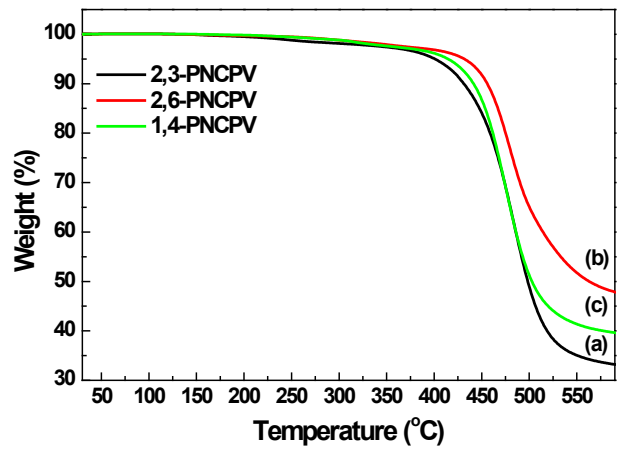

Fig. 2. TGA thermograms of (a) 2,3-PNCPV, (b) 2,6-PNCPV, and (c) 1,4-PNCPV.

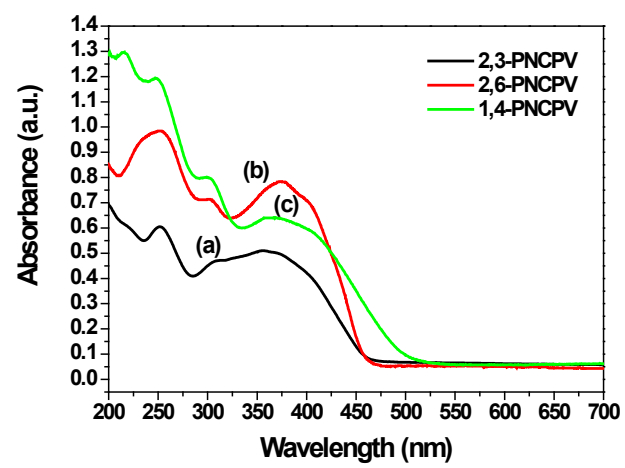

Fig. 3. UV-visible spectra of (a) 2,3-PNCPV, (b) 2,6-PNCPV, and (c) 1,4-PNCPV thin films coated on a quartz plate.

in PMMA film at a concentration of less than $10^{-3} \mathrm{M}$, assuming a PL efficiency of $\Phi_{\text {film }}=0.83$ ) [15]. 2,3-, 2,6- and 1,4-PNCPV showed relatively higher PL efficiencies of $\Phi_{\text {film }}=0.71,0.69$, and 0.59. 2,3-PNCPV and 2,6-PNCPV exhibited higher PL efficiencies than 1,4-PNCPV both in solution and film states. The polymer main-chain packing and conjugation are more effectively inter- 


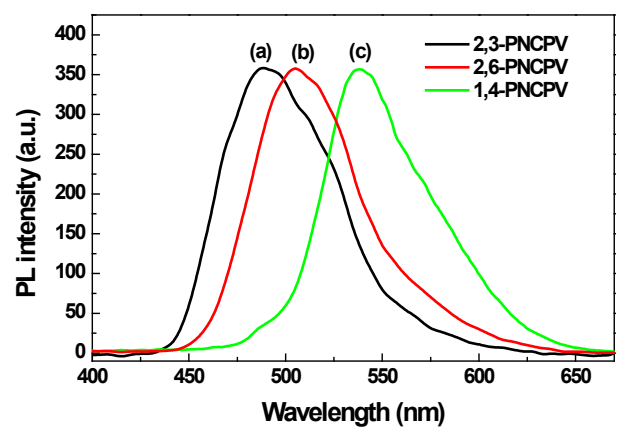

Fig. 4. Photoluminescence spectra of (a) 2,3-PNCPV, (b) 2,6-PNCPV, and (c) 1,4-PNCPV thin films coated on a quartz plate.

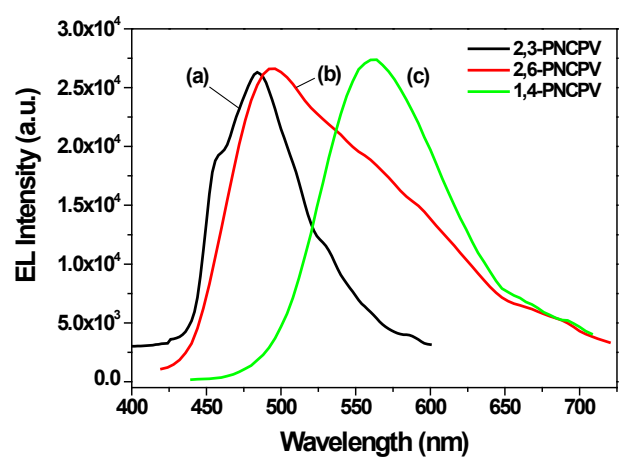

Fig. 5. Electroluminescence spectra of the single-layer light-emitting diodes of (a) 2,3-PNCPV, (b) 2,6-PNCPV, and (c) 1,4-PNCPV which have ITO/polymer/Al configuration.

rupted in the 2,3- or 2,6-linked naphthalene systems (2,3-PNCPV and 2,6-PNCPV) than the 1,4-linked naphthalene system (1,4-PNCPV), which results in the formation of amorphous polymer thin films for increased luminescence.

A single-layer light-emitting diode was fabricated using ITO as the anode and $\mathrm{Al}$ as the cathode. The 2,3-PNCPV, 2,6-PNCPV or 1,4-PNCPV polymers were deposited onto indium-tin oxidecovered glass substrates by spin-casting the soluble polymers in cyclohexanone. The spin-casting technique yielded uniform films with a thicknesses of about $70 \sim 80 \mathrm{~nm}$. The Al electrode (cathode) was then evaporated onto this in vacuo with thickness of about $100 \mathrm{~nm}$. The EL spectra of 2,3-PNCPV, 2,6-PNCPV and 1,4-PNCPV polymer films are shown in Figure 5. The 1,4-PNCPV polymer film showed maximum EL emission at $560 \mathrm{~nm}$, which was somewhat red-shifted compared to that of the PL spectrum of 1,4-PNCPV. The 2,6-PNCPV exhibited EL maximum emissive bands at $494 \mathrm{~nm}$ with broad spectrum bandwidth. As expected from the UV-visible absorption and PL data, 2,3-PNCPV showed almost pure blue EL emission at $483 \mathrm{~nm}$.

This result clearly means that the $\pi$-conjugation length could be systematically controlled by the 2,3-linked naphthalene group, enabling blue EL emission. These results indicate that naphthalene and carbazole containing PPV derivatives could be good candidates for blue-emitting material. Figure 6(a) shows the current vs. voltage characteristics measured from the synthesized polymers. The forward current increases with increasing forward bias voltage for all devices. The threshold voltages of all polymers were in the range of 7 to $13 \mathrm{~V}$. Figure 6(b) exhibits the EL intensity vs. current characteristics from 2,3-PNCPV, 2,6-PNCPV and 1,4-PNCPV devices, respectively. The EL efficiency of 2,3-PNCPV with a 2,3-linked naphthalene unit shows the (a)

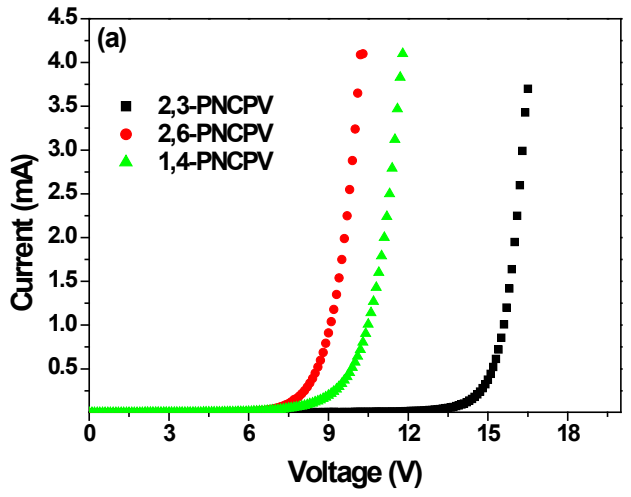

(b)

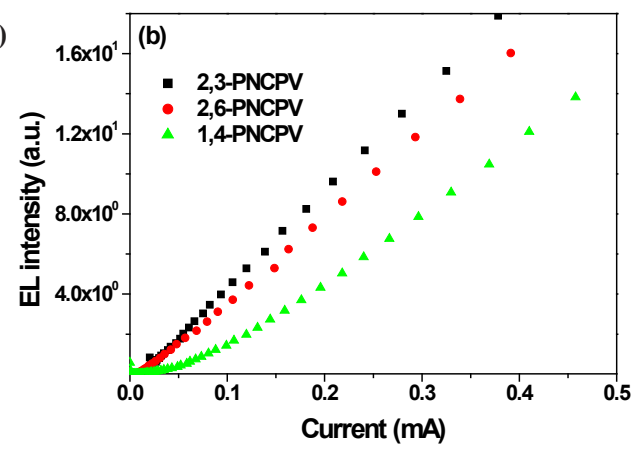

Fig. 6. (a) Current vs. voltage characteristics and (b) EL intensity vs. current characteristics of 2,3-PNCPV, 2,6-PNCPV and 1,4-PNCPV polymers.

highest efficiency compared to polymers with 2,6- or 1,4-linked naphthalene groups at the same current density. Further device optimizations and electrical characterizations of the synthesized polymers are in progress by replacing the cathode electrode with $\mathrm{LiF} / \mathrm{Al}$ or $\mathrm{Ca} / \mathrm{Al}$ or by the insertion of a PEDOT layer as a hole injection layer to increase the EL efficiency of the synthesized polymers.

\section{CONCLUSIONS}

Differently linked naphthalene and carbazole-containing 2,3-PNCPV, 2,6-PNCPV and 1,4-PNCPV polymers have been synthesized through the Wittig condensation polymerization. The $\pi$-conjugation lengths of synthesized polymers were regulated by 2,3-, 2,6- or 1.4-linked naphthalene groups. All polymers were completely soluble in organic solvents and could be made into homogeneous thin films without heat treatment. In the UV-visible and PL spectra, the maximum absorption and PL emission spectra were systematically blue-shifted because of twisted 2,3or 2,6-linked naphthalene and carbazole groups. Consequently 2,3-PNCPV showed blue PL emission at $487 \mathrm{~nm}$. A single-layer light-emitting diode of ITO/2,3-PNCPV/Al was fabricated. The EL spectrum of 2,3-PNCPV gave the highest peak at $483 \mathrm{~nm}$, which corresponds to pure blue emission. It is proposed that the carbazole-containing PPV derivatives linked by the naphthalene group could be promising candidates for blue-emitting polymers.

\section{ACKNOWLEDGMENTS}

This research was supported by Kyungsung University Research Grants in 2013. 


\section{REFERENCES}

[1] J. H. Burroughs, D. D. C. Bradley, A. R. Brown, R. N. Marks, K. Mackay, R. H. Friend, P. L. Burn, and A. B. Holmes, Nature 347, 539 (1990) [DOI: http://dx.doi.org/ 10.1038/347539a0].

[2] D. Braun, and A. J. Heeger, Appl. Phys. Lett. 58, 1982 (1991) [DOI: http://dx.doi.org/10.1063/1.105039].

[3] C. W. Tang and S. A. VanSlyke, Appl. Phys. Lett. 51, 913 (1987) [DOI: http://dx.doi.org/10.1063/1.98799].

[4] G. Gustafasson, Y. Cao, G. M. Treacy, F. Klavetter, N. Colaneri and A. J. Heeger, Nature 357, 477 (1992) [DOI: http://dx.doi org/10.1038/357477a0].

[5] D. D. C. Bradley, Synth. Met. 54, 401 (1993) [DOI: http://dx.doi. org/10.1016/0379-6779(93)91086-H].

[6] G. Grem, G. Leditzky, B. Ullrich, and G. Leising, Adv. Mater. 4, 36 (1992) [DOI: http://10.1002/adma.19920040107].

[7] R. D. Miller and G. Klaerner, Macromolecules 31, 2007 (1998) [DOI: http://dx.doi.org/10.1021/ma971073e].

[8] I. Sokolik, Z. Yang, F. E. Karasaz, and D. C. Morton, J. Appl. Phys.
74, 3584 (1993) [DOI: http://dx.doi.org/10.1063/1.354539].

[9] T. Ahn, M. S. Jang, H. K. Shim, D. H. Hwang, and T. Zyung, Macromolecules 32, 3279 (1999) [DOI: http://dx.doi.org/10.1021/ ma981864w]

[10] T. Ahn, S. Y. Song, and H. K. Shim, Macromolecules 33, 6764, 2000 [DOI: http://dx.doi.org/10.1021/ma000449c].

[11] T. Ahn, Trans. Electr. Electron. Mater. 13, 317 (2012) [DOI: http://dx.doi.org/10.4313/TEEM.2012.13.6.317].

[12] P. L. burn, A. Kraft, D. R. Baigent, D. D. C. Bradley, A. R. Brown, R. H. Friend, R. W. Gymer, A. B. Holmes, R. W. Jackson, J. Am. Chem. Soc. 115, 10117 (1993) [DOI: http://10.1021/ ja00075a030].

[13] H. W. Kim, M. K. Ryu, S. M. Lee, Macromolecules 30, 1236 (1997) [DOI: http://10.1021/ma961295x].

[14] H. S. Joshi, R. Jamshidi, Y. Tor, Angew. Chem., Int. Ed. 38, 2721 (1999) [DOI: http://10.1002/(SICI)15213773(19990917)38:18<2721::AID-ANIE2721>3.0.CO;2-5].

[15] H. Meng, W. L. Yu, W. Huang, Macromolecules 32, 8841 (1999) [DOI: http://10.1021/ma9907439]. 